PROCEEDINGS OF THE

AMERICAN MATHEMATICAL SOCIETY

Volume 135, Number 8, August 2007, Pages 2369-2380

S 0002-9939(07)08975-7

Article electronically published on April 10, 2007

\title{
ASYMPTOTICS OF BEST-PACKING ON RECTIFIABLE SETS
}

\author{
S. V. BORODACHOV, D. P. HARDIN, AND E. B. SAFF
}

(Communicated by David Preiss)

\begin{abstract}
We investigate the asymptotic behavior, as $N$ grows, of the largest minimal pairwise distance of $N$ points restricted to an arbitrary compact rectifiable set embedded in Euclidean space, and we find the limit distribution of such optimal configurations. For this purpose, we compare best-packing configurations with minimal Riesz s-energy configurations and determine the $s$-th root asymptotic behavior (as $s \rightarrow \infty$ ) of the minimal energy constants.

We show that the upper and the lower dimension of a set defined through the Riesz energy or best-packing coincides with the upper and lower Minkowski dimension, respectively.

For certain sets in $\mathbf{R}^{d}$ of integer Hausdorff dimension, we show that the limiting behavior of the best-packing distance as well as the minimal $s$-energy for large $s$ is different for different subsequences of the cardinalities of the configurations.
\end{abstract}

\section{Preliminaries}

The problem of finding a configuration of $N$ points on the sphere with the minimal pairwise distance between the points being as large as possible is classical and is known as Tammes's problem or the hard spheres problem. When formulated for the whole Euclidean space, the analogous problem is that of finding a collection (or packing) of non-overlapping equal balls with the largest density. More information on this problem and its generalizations can be found in [2], 4], 7], 19]. In the present paper we investigate the best-packing problem on certain classes of "non-smooth" sets.

1.1. Best-packing problem. We denote by $\mathbf{R}^{d^{\prime}}$ the embedding space, reserving the symbol $d$ for the dimension of the set being considered. For a collection of $N$ distinct points $\omega_{N}=\left\{y_{1}, \ldots, y_{N}\right\} \subset \mathbf{R}^{d^{\prime}}$ we set

$$
\delta\left(\omega_{N}\right):=\min _{1 \leq i \neq j \leq N}\left|y_{i}-y_{j}\right|
$$

Received by the editors April 19, 2006.

2000 Mathematics Subject Classification. Primary 11K41, 70F10, 28A78; Secondary 78A30, $52 \mathrm{~A} 40$.

Key words and phrases. Best-packing points, sphere packing, rectifiable set, Thomson problem, packing measure, minimal discrete Riesz energy, hard spheres problem.

The research of the second author was supported, in part, by the U. S. National Science Foundation under grants DMS-0505756 and DMS-0532154.

The research of the third author was supported, in part, by the U. S. National Science Foundation under grant DMS-0532154.

(C)2007 American Mathematical Society Reverts to public domain 28 years from publication 
and for an infinite set $A \subset \mathbf{R}^{d^{\prime}}$, we let

$$
\delta_{N}(A):=\sup \left\{\delta\left(\omega_{N}\right): \omega_{N} \subset A, \# \omega_{N}=N\right\}
$$

be the best-packing distance of $N$-point configurations on $A$, where $\# X$ denotes the cardinality of the set $X$. We can consider only compact infinite sets $A$, since quantity (1.1) is infinite for unbounded sets and is the same for a set and its closure.

For the case when $A$ is the unit sphere $S^{2} \subset \mathbf{R}^{3}$, exact values of $\delta_{N}(A)$ have been determined for $2 \leq N \leq 12$ and $N=24$ (see [2] for references). For arbitrarily large values of $N$, the precise determination of best-packing distances is, in general, an intractable problem. Thus we focus on their asymptotic behavior. For this purpose we introduce the following notation. Let $0<\alpha \leq d^{\prime}$ and set

$$
\underline{g}_{\infty, \alpha}(A):=\liminf _{N \rightarrow \infty} \delta_{N}(A) \cdot N^{1 / \alpha}, \quad \bar{g}_{\infty, \alpha}(A):=\limsup _{N \rightarrow \infty} \delta_{N}(A) \cdot N^{1 / \alpha} .
$$

We further put

$$
g_{\infty, \alpha}(A):=\lim _{N \rightarrow \infty} \delta_{N}(A) \cdot N^{1 / \alpha},
$$

if this limit exists. On relating these quantities to the largest sphere packing density in $\mathbf{R}^{d}, d \in \mathbf{N}$, which we denote by $\Delta_{d}$ (see (1.4) below), it can be shown that $g_{\infty, d}\left([0,1]^{d}\right)$ exists and is given by

$$
C_{\infty, d}:=g_{\infty, d}\left([0,1]^{d}\right)=2\left(\Delta_{d} / \beta_{d}\right)^{1 / d},
$$

where $\beta_{d}$ is the Lebesgue measure (volume) of the unit ball in $\mathbf{R}^{d}$. It is not difficult to show that $g_{\infty, d}(A)$ exists for $d$-dimensional smooth manifolds and domains. Here we shall establish the existence of $g_{\infty, d}(A)$ for a class of rectifiable sets and provide a formula for it in terms of the largest sphere packing density in $\mathbf{R}^{d}$; we also describe the limiting distribution of best-packing points (see Theorem [2.2).

Recall that the definition of $\Delta_{d}$ is as follows (cf. [6, Chapter 3] or [19, Chapter 1]). Let $\mathcal{L}_{d}$ stand for the Lebesgue measure in $\mathbf{R}^{d}$. Denote by $\Lambda_{d}$ the set of collections $\mathcal{P}$ of non-overlapping unit balls in $\mathbf{R}^{d}$ for which the density

$$
\rho(\mathcal{P}):=\lim _{r \rightarrow \infty}(2 r)^{-d} \cdot \mathcal{L}_{d}\left(\bigcup_{B \in \mathcal{P}} B \cap[-r, r]^{d}\right)
$$

exists. Then

$$
\Delta_{d}:=\sup _{\mathcal{P} \in \Lambda_{d}} \rho(\mathcal{P}) .
$$

It is known that $\Delta_{1}=1, \Delta_{2}=\pi / \sqrt{12}$ (Thue in 1892 and Fejes-Toth [6]), and $\Delta_{3}=\pi / \sqrt{18}$ (Hales [10]). The exact value of $\Delta_{d}$ for $d>3$ is unknown; so far, only upper and lower estimates, which differ by an exponential factor as $d \rightarrow \infty$, have been obtained for $\Delta_{d}$ (see [4] for this and other references).

Concerning the case when $A=S^{2}$, the papers [13, 21] prove that $g_{\infty, 2}\left(S^{2}\right)=$ $(8 \pi / \sqrt{3})^{1 / 2}$. Furthermore, the results of [10] imply that for the unit sphere $S^{3} \subset \mathbf{R}^{4}$ we have $g_{\infty, 3}\left(S^{3}\right)=\sqrt{2} \pi^{2 / 3}$.

1.2. Minimum energy problem. The best-packing problem can be viewed as the limiting case of the problem of minimization of the discrete Riesz $s$-energy as 
$s \rightarrow \infty$. The setting of this latter problem is as follows: for $s>0$ and a collection $\omega_{N}=\left\{y_{1}, \ldots, y_{N}\right\} \subset \mathbf{R}^{d^{\prime}}$, let

$$
E_{s}\left(\omega_{N}\right):=\sum_{i=1}^{N} \sum_{j: j \neq i} \frac{1}{\left|y_{i}-y_{j}\right|^{s}} .
$$

The minimal discrete $N$-point Riesz s-energy of a set $A$ is defined to be

$$
\mathcal{E}_{s}(A, N):=\inf \left\{E_{s}\left(\omega_{N}\right): \omega_{N} \subset A, \# \omega_{N}=N\right\} .
$$

It is not difficult to see that, for $N$ fixed, $\lim _{s \rightarrow \infty} \mathcal{E}_{s}(A, N)^{1 / s}=1 / \delta_{N}(A)$. We remark that for the case when $s=1$ and $A=S^{2}$, the determination of $\mathcal{E}_{s}(A, N)$ corresponds to the famous Thomson problem for the Coulomb potential (cf. 20]). Exact solutions to this problem are known for $N=2,3,4,6,12$ and for some interesting cases in higher dimensions (see 22], 15], 1] and the references therein).

When $0<s<n:=\operatorname{dim}_{H} A$ (the Hausdorff dimension of the set $A$ ), potential theory implies that $\mathcal{E}_{s}(A, N) \asymp N^{2}, N \rightarrow \infty$ (cf. e.g. [16]). When $s>n$, local interactions dominate, and as described in Theorem 2.1 below, $\mathcal{E}_{s}(A, N) \asymp N^{1+s / n}$, $N \rightarrow \infty$, for compact rectifiable sets $A$ of positive $n$-dimensional Hausdorff measure.

For analogous asymptotic results concerning optimal quantization of measures see [9] and the references therein.

1.3. Notation and definitions. To describe the precise rate of growth of $\mathcal{E}_{s}(A, N)$, where $A$ may have non-integer dimension, let $0<\alpha \leq d^{\prime}$ and for $s>\alpha$ define

$$
\underline{g}_{s, \alpha}(A):=\liminf _{N \rightarrow \infty} \frac{\mathcal{E}_{s}(A, N)}{N^{1+s / \alpha}}, \quad \bar{g}_{s, \alpha}(A):=\limsup _{N \rightarrow \infty} \frac{\mathcal{E}_{s}(A, N)}{N^{1+s / \alpha}}
$$

and

$$
g_{s, \alpha}(A):=\lim _{N \rightarrow \infty} \frac{\mathcal{E}_{s}(A, N)}{N^{1+s / \alpha}}
$$

if this limit exists. Given a positive integer $d \leq d^{\prime}$, denote by $\mathcal{H}_{d}$ the $d$-dimensional Hausdorff measure in $\mathbf{R}^{d^{\prime}}$ normalized so that an isometric image of $[0,1]^{d}$ has measure 1. Following [5] a set $A \subset \mathbf{R}^{d^{\prime}}$ is called d-rectifiable if it is an image of a bounded set from $\mathbf{R}^{d}$ with respect to a Lipschitz mapping. A set $A \subset \mathbf{R}^{d^{\prime}}$ is called $\left(\mathcal{H}_{d}, d\right)$-rectifiable, if $\mathcal{H}_{d}(A)<\infty$ and $A$ is a union of at most a countable collection of $d$-rectifiable sets and a set of $\mathcal{H}_{d}$-measure zero.

Given $\alpha \geq 0$, let $\beta_{\alpha}=\frac{\pi^{\alpha / 2}}{\Gamma(1+\alpha / 2)}$. When $\alpha$ is an integer, this formula agrees with the above definition of $\beta_{d}$. Also let $A(\epsilon), \epsilon>0$, be the $\epsilon$-neighborhood of the set $A \subset \mathbf{R}^{d^{\prime}}$. The lower and the upper Minkowski content of $A$ are defined by

$$
\underline{\mathcal{M}}_{\alpha}(A):=\liminf _{\rho \rightarrow 0^{+}} \frac{\mathcal{L}_{d^{\prime}}(A(\rho))}{\beta_{d^{\prime}-\alpha} \rho^{d^{\prime}-\alpha}} \quad \text { and } \quad \overline{\mathcal{M}}_{\alpha}(A):=\limsup _{\rho \rightarrow 0^{+}} \frac{\mathcal{L}_{d^{\prime}}(A(\rho))}{\beta_{d^{\prime}-\alpha} \rho^{d^{\prime}-\alpha}},
$$

respectively. If they coincide, then the quantity $\mathcal{M}_{\alpha}(A):=\underline{\mathcal{M}}_{\alpha}(A)=\overline{\mathcal{M}}_{\alpha}(A)$ is called the $\alpha$-dimensional Minkowski content of the set $A$. It is known (cf. 5, Theorem 3.2.39]) that for every closed $d$-rectifiable set $A \subset \mathbf{R}^{d^{\prime}}$ we have

$$
\mathcal{M}_{d}(A)=\mathcal{H}_{d}(A)
$$

Let $A$ be compact with $\mathcal{H}_{d}(A)>0$ and $\left\{\omega_{N}\right\}_{N=2}^{\infty}$ be a sequence of point configurations on $A$ such that $\# \omega_{N}=N, N \geq 2$. We say that $\left\{\omega_{N}\right\}_{N=2}^{\infty}$ is asymptotically 
uniformly distributed on $A$ with respect to $\mathcal{H}_{d}$ if, for every subset $B \subset A$ whose boundary relative to $A$ has $\mathcal{H}_{d}$-measure zero, we have

$$
\frac{\#\left(\omega_{N} \cap B\right)}{N} \rightarrow \frac{\mathcal{H}_{d}(B)}{\mathcal{H}_{d}(A)}, \quad N \rightarrow \infty .
$$

This definition can also be stated in terms of the weak* convergence of measures.

\section{MAin Results}

First, we describe known results on the asymptotic behavior of the minimal $s$-energy on sets with $\operatorname{dim}_{H} A=d$ and $s>d$. Let

$$
C_{s, d}:=\lim _{N \rightarrow \infty} \frac{\mathcal{E}_{s}\left([0,1]^{d}, N\right)}{N^{1+s / d}}, s>d .
$$

In [17] it is shown that $C_{s, 1}=2 \zeta(s), s>1$, where $\zeta(s)=\sum_{i=1}^{\infty} i^{-s}$ is the classical zeta-function. The articles [11, [12] show that for $s>d$ the limit $C_{s, d}$ exists, is finite and is positive. However, the value of $C_{s, d}$ is still unknown for $d>1$.

The following result is proved in [12, 3] (for curves in $\mathbf{R}^{d^{\prime}}$, it follows from [17]).

Theorem 2.1. Let $s>d$ and $d^{\prime} \geq d$, where $d$ and $d^{\prime}$ are integers. For every infinite compact $\left(\mathcal{H}_{d}, d\right)$-rectifiable set $A$ in $\mathbf{R}^{d^{\prime}}$ with $\mathcal{M}_{d}(A)=\mathcal{H}_{d}(A)$, the limit $g_{s, d}(A)$ defined in (1.7) exists and is given by

$$
g_{s, d}(A)=C_{s, d} \mathcal{H}_{d}(A)^{-s / d} .
$$

Moreover, if $A$ is d-rectifiable with $\mathcal{H}_{d}(A)>0$, then any sequence $\left\{\omega_{N}^{*}\right\}_{N=2}^{\infty}$ of $s$ energy minimizing collections on $A$ such that $\# \omega_{N}^{*}=N$ is asymptotically uniformly distributed on $A$ with respect to $\mathcal{H}_{d}$.

In the case $d=d^{\prime}$, Theorem 2.1 applies to any compact set $A \subset \mathbf{R}^{d^{\prime}}$, since such a set is trivially $d$-rectifiable. In this case $\mathcal{H}_{d}=\mathcal{L}_{d^{\prime}}$, but we use the notation $\mathcal{H}_{d}$ to handle both the cases $d<d^{\prime}$ and $d=d^{\prime}$.

In this paper we get an analogue of Theorem 2.1 for best-packing configurations.

Theorem 2.2. Let $d \leq d^{\prime}$, where $d, d^{\prime}$ are integers, and $A \subset \mathbf{R}^{d^{\prime}}$ be an infinite compact $\left(\mathcal{H}_{d}, d\right)$-rectifiable set. If $\mathcal{M}_{d}(A)=\mathcal{H}_{d}(A)$, then $g_{\infty, d}(A)$ exists and is given by

$$
g_{\infty, d}(A)=C_{\infty, d} \cdot \mathcal{H}_{d}(A)^{1 / d}=2\left(\Delta_{d} / \beta_{d}\right)^{1 / d} \cdot \mathcal{H}_{d}(A)^{1 / d} .
$$

Moreover, if $\overline{\mathcal{M}}_{d}(A)>\mathcal{H}_{d}(A)$, then

$$
\bar{g}_{\infty, d}(A)>C_{\infty, d} \cdot \mathcal{H}_{d}(A)^{1 / d} .
$$

If $A$ is d-rectifiable with $\mathcal{H}_{d}(A)>0$, then every sequence $\left\{\bar{\omega}_{N}\right\}_{N=2}^{\infty}$ of best-packing configurations on $A$ such that $\# \bar{\omega}_{N}=N$ is asymptotically uniformly distributed on A with respect to $\mathcal{H}_{d}$.

In view of relation (1.9), and the fact that any $\left(\mathcal{H}_{d}, d\right)$-rectifiable set can be approximated by its $d$-rectifiable subsets, we either have $\mathcal{M}_{d}(A)=\mathcal{H}_{d}(A)$ or $\overline{\mathcal{M}}_{d}(A)>\mathcal{H}_{d}(A)$, so that either (2.3) or (2.4) must hold.

For results similar to (2.3) for $d=d^{\prime}$ that concern the covering radius, see 8 .

We next relate the fundamental constants $C_{s, d}$ and $C_{\infty, d}$. 
Theorem 2.3. The limit $\lim _{s \rightarrow \infty} C_{s, d}^{1 / s}$ exists for each integer $d>1$ and

$$
\lim _{s \rightarrow \infty} C_{s, d}^{1 / s}=\frac{1}{C_{\infty, d}}=\frac{1}{2}\left(\frac{\beta_{d}}{\Delta_{d}}\right)^{1 / d} .
$$

We next show that relation (2.2) can fail for certain $\left(\mathcal{H}_{d}, d\right)$-rectifiable sets.

Proposition 2.4. If $A \subset \mathbf{R}^{d^{\prime}}$ is a compact $\left(\mathcal{H}_{d}, d\right)$-rectifiable set with $\overline{\mathcal{M}}_{d}(A)>$ $\mathcal{H}_{d}(A)$, then for s sufficiently large

$$
\underline{g}_{s, d}(A)<C_{s, d} \mathcal{H}_{d}(A)^{-s / d} .
$$

As an example of a rectifiable set for which (1.9) does not hold, we mention a compact $\left(\mathcal{H}_{2}, 2\right)$-rectifiable set $B \subset \mathbf{R}^{3}$ with $0<\mathcal{H}_{2}(B)<\infty=\mathcal{M}_{2}(B)$ given in [5. p. 276]. Proposition 2.5 will imply that $g_{s, 2}(B)=0, s>3$, and $g_{\infty, 2}(B)=\infty$.

Remarks for general sets. Let $\underline{\operatorname{dim}}_{M} A$ and $\overline{\operatorname{dim}}_{M} A$ denote the lower and the upper Minkowski dimension of a set $A \subset \mathbf{R}^{d^{\prime}}$. One can also introduce the lower and the upper dimension of a set using $s$-energy or best-packing. Let $\underline{\operatorname{dim}}_{\infty} A:=\inf (\{\alpha>$ $\left.\left.0: \underline{g}_{\infty, \alpha}(A)=0\right\} \cup\left\{d^{\prime}\right\}\right)=\sup \left(\left\{\alpha \in\left(0, d^{\prime}\right]: \underline{g}_{\infty, \alpha}(A)=\infty\right\} \cup\{0\}\right)$ and for a fixed $s>d^{\prime}$ denote $\underline{\operatorname{dim}}_{s} A:=\inf \left(\left\{\alpha>0: \bar{g}_{s, \alpha}(A)=\infty\right\} \cup\left\{d^{\prime}\right\}\right)=\sup \left(\left\{\alpha \in\left(0, d^{\prime}\right]:\right.\right.$ $\left.\left.\bar{g}_{s, \alpha}(A)=0\right\} \cup\{0\}\right)$ with $\overline{\operatorname{dim}}_{\infty} A$ and $\overline{\operatorname{dim}}_{s} A$ being defined in an analogous way through $\bar{g}_{\infty, \alpha}$ or $\underline{g}_{s, \alpha}$. The following proposition implies that for any set $A \subset \mathbf{R}^{d^{\prime}}$ we have $\underline{\operatorname{dim}}_{s} A=\underline{\operatorname{dim}}_{\infty} A=\underline{\operatorname{dim}}_{M} A$ and $\overline{\operatorname{dim}}_{s} A=\overline{\operatorname{dim}}_{\infty} A=\overline{\operatorname{dim}}_{M} A$, provided $s>d^{\prime}$.

Proposition 2.5. If $0<\alpha \leq d^{\prime}<s$, there are positive constants $c_{1}=c_{1}(s, \alpha)$ and $c_{2}=c_{2}(s, \alpha)$ such that for any infinite set $A \subset \mathbf{R}^{d^{\prime}}$ we have

$$
\begin{aligned}
& c_{1} \underline{\mathcal{M}}_{\alpha}(A)^{-s / \alpha} \leq \bar{g}_{s, \alpha}(A) \leq c_{2} \underline{\mathcal{M}}_{\alpha}(A)^{-s / \alpha}, \\
& c_{1} \overline{\mathcal{M}}_{\alpha}(A)^{-s / \alpha} \leq \underline{g}_{s, \alpha}(A) \leq c_{2} \overline{\mathcal{M}}_{\alpha}(A)^{-s / \alpha} .
\end{aligned}
$$

There are also positive constants $c_{3}=c_{3}(\alpha)$ and $c_{4}=c_{4}(\alpha)$ such that for every infinite set $A \subset \mathbf{R}^{d^{\prime}}$

$$
\begin{aligned}
& c_{3} \underline{\mathcal{M}}_{\alpha}(A)^{1 / \alpha} \leq \underline{g}_{\infty, \alpha}(A) \leq c_{4} \underline{\mathcal{M}}_{\alpha}(A)^{1 / \alpha}, \\
& c_{3} \overline{\mathcal{M}}_{\alpha}(A)^{1 / \alpha} \leq \bar{g}_{\infty, \alpha}(A) \leq c_{4} \overline{\mathcal{M}}_{\alpha}(A)^{1 / \alpha} .
\end{aligned}
$$

It is known that $\operatorname{dim}_{M} A \geq \operatorname{dim}_{H} A$ with a strict inequality possible for some compact sets (cf. e.g. [18, p. 77]). Hence, for such sets $A$ and any $\operatorname{dim}_{H} A<\alpha<$ $\alpha_{1}<\underline{\operatorname{dim}}_{M} A$ we have $\mathcal{H}_{\alpha}(A)=0$, but $g_{s, \alpha_{1}}(A)=0, s>d^{\prime}$, and $g_{\infty, \alpha_{1}}(A)=\infty$.

For every $s \in\left(d^{\prime}, \infty\right]$ and compact sets with sufficiently large gap between $\underline{\mathcal{M}}_{\alpha}(A)$ and $\overline{\mathcal{M}}_{\alpha}(A)$ we will have $\underline{g}_{s, \alpha}(A)<\bar{g}_{s, \alpha}(A)$. Moreover, if $\underline{\operatorname{dim}}_{M} A<$ $\overline{\operatorname{dim}}_{M} A$ (cf. e.g. [18, p. 77] for examples), the order of the best-packing radius and the minimal $s$-energy for $s>d^{\prime}$ will vary depending on the subsequence of cardinalities of configurations.

We also show that the condition of $\left(\mathcal{H}_{d}, d\right)$-rectifiability in Theorems 2.1 and 2.2 is crucial in the sense that there are non-rectifiable compact sets with $\operatorname{dim}_{H} A=d$ and $0<\mathcal{H}_{d}(A)<\infty$ such that $g_{\infty, d}(A)$ and $g_{s, d}(A)$ (for sufficiently large $s$ ) do not 
exist. Indeed, we show that this is true for a class of Cantor-type sets which we will denote by $\mathcal{K}$.

We say that a non-empty compact set $K \subset \mathbf{R}^{d^{\prime}}$ belongs to the class $\mathcal{K}$, if there are a finite number of distinct similitudes $S_{1}, \ldots, S_{p}: \mathbf{R}^{d^{\prime}} \rightarrow \mathbf{R}^{d^{\prime}}$ with the same contraction coefficient $\sigma \in(0,1)$ such that

$$
\bigcup_{i=1}^{p} S_{i}(K)=K, \quad \text { and } \quad S_{i}(K) \cap S_{j}(K)=\emptyset, \quad i \neq j .
$$

According to [14, we have $\lambda:=\operatorname{dim}_{H} K=-\log _{\sigma} p$ and $0<\mathcal{H}_{\lambda}(K)<\infty$. This is a subclass of the class of self-similar sets constructed in [14] (this construction is also cited in [18, Section 4.13]).

Class $\mathcal{K}$ contains the classical Cantor subset of $[0,1]$. Parameters $p$ and $\sigma$ can also be chosen so that $\operatorname{dim}_{H} K$ is any integer between 0 and $d^{\prime}$. For example, if $a_{1}$, $a_{2}$, and $a_{3}$ are vertices of an equilateral triangle on the plane and $S_{i}, i=1,2,3$, is the homothety of the plane with respect to $a_{i}$ and the contraction coefficient $1 / 3$, then we get a set of Hausdorff dimension one, known as the Sierpinski gasket [18, p. 75$]$.

Proposition 2.6. Let $K$ be a compact set from the class $\mathcal{K}$ with $\lambda=\operatorname{dim}_{H} K$. Then, for s sufficiently large we have $0<\underline{g}_{s, \lambda}(K)<\bar{g}_{s, \lambda}(K)<\infty$.

\section{Proofs}

With regard to the extended real number limits in $[0, \infty]$, we agree that $1 / 0=$ $0^{-s}=\infty^{s}=\infty, 1 / \infty=\infty^{-s}=0, s>0$.

Proposition 3.1. For every infinite set $A \subset \mathbf{R}^{d^{\prime}}$ and $0<\alpha \leq d^{\prime}$ we have

$$
\lim _{s \rightarrow \infty}\left(\bar{g}_{s, \alpha}(A)\right)^{1 / s}=\frac{1}{\underline{g}_{\infty, \alpha}(A)} \text { and } \lim _{s \rightarrow \infty}\left(\underline{g}_{s, \alpha}(A)\right)^{1 / s}=\frac{1}{\bar{g}_{\infty, \alpha}(A)} .
$$

Proposition 3.1 immediately yields the following statements.

Proposition 3.2. Let $A \subset \mathbf{R}^{d^{\prime}}$ be an infinite set and $0<\alpha \leq d^{\prime}$. If for every $s$ sufficiently large $\underline{g}_{s, \alpha}(A)=\bar{g}_{s, \alpha}(A)$, then $g_{\infty, \alpha}(A)$ exists and

$$
\lim _{s \rightarrow \infty}\left(g_{s, \alpha}(A)\right)^{1 / s}=\frac{1}{g_{\infty, \alpha}(A)} .
$$

Proposition 3.3. Let $A \subset \mathbf{R}^{d^{\prime}}$ be an infinite set such that $\underline{g}_{\infty, \alpha}(A)<\bar{g}_{\infty, \alpha}(A)$ for some $0<\alpha \leq d^{\prime}$. Then for sufficiently large $s$ we have $\underline{g}_{s, \alpha}(A)<\bar{g}_{s, \alpha}(A)$.

Proof of Proposition 3.1. Lower estimates. We can assume $A \subset \mathbf{R}^{d^{\prime}}$ to be compact, since on unbounded sets $g_{s, \alpha}(A)=0$ and $g_{\infty, \alpha}(A)=\infty$ and the minimal $s$-energy (as well as the best-packing radius) is the same for $A$ and its closure.

Choose an arbitrary $\epsilon \in(0,1)$ and let $s>\alpha$. Let $N$ be sufficiently large and $\omega_{N}^{*}:=\left\{x_{1, N}, \ldots, x_{N, N}\right\}$ be an $s$-energy minimizing $N$-point collection on $A$. Set $N_{\epsilon}:=\lfloor(1-\epsilon) N\rfloor$, where $\lfloor t\rfloor$ is the floor function of $t$, and

$$
r_{i, N}:=\min _{j: j \neq i}\left|x_{i, N}-x_{j, N}\right| .
$$


Pick a point $x_{i_{1}, N} \in \omega_{N}^{*}$ with $r_{i_{1}, N} \leq \delta_{N}(A)$. In $\omega_{N}^{*} \backslash\left\{x_{i_{1}, N}\right\}$ pick a point $x_{i_{2}, N}$ so that $r_{i_{2}, N} \leq \delta_{N-1}(A)$. Continue this process until we pick a point $x_{i_{\lfloor\in N\rfloor+1}, N} \in$ $\omega_{N}^{*} \backslash\left\{x_{i_{1}, N}, \ldots, x_{i_{\lfloor\in N\rfloor}, N}\right\}$ such that $r_{i_{\lfloor\epsilon N\rfloor+1}, N} \leq \delta_{N-\lfloor\epsilon N\rfloor}(A)$. Then

$$
\mathcal{E}_{s}(A, N)=E_{s}\left(\omega_{N}^{*}\right) \geq \sum_{k=1}^{\lfloor\epsilon N\rfloor+1} \frac{1}{\left(r_{i_{k}, N}\right)^{s}} \geq \sum_{k=1}^{\lfloor\epsilon N\rfloor+1} \frac{1}{\left(\delta_{N-k+1}(A)\right)^{s}} \geq \frac{\epsilon N}{\left(\delta_{N_{\epsilon}}(A)\right)^{s}} .
$$

Hence,

$$
\bar{g}_{s, \alpha}(A) \geq \limsup _{N \rightarrow \infty} \frac{\epsilon}{\left(\delta_{N_{\epsilon}}(A)\right)^{s} N^{s / \alpha}}=\frac{\epsilon(1-\epsilon)^{s / \alpha}}{\left(\liminf _{N \rightarrow \infty} \delta_{N_{\epsilon}}(A) \cdot N_{\epsilon}^{1 / \alpha}\right)^{s}}=\frac{\epsilon(1-\epsilon)^{s / \alpha}}{\left(\underline{g}_{\infty, \alpha}(A)\right)^{s}},
$$

since $N_{\epsilon}$ passes through all natural numbers. Similarly,

$$
\underline{g}_{s, \alpha}(A) \geq \frac{\epsilon(1-\epsilon)^{s / \alpha}}{\left(\bar{g}_{\infty, \alpha}(A)\right)^{s}}
$$

Then, first letting $s \rightarrow \infty$ and then $\epsilon \rightarrow 0$, we get

$$
\liminf _{s \rightarrow \infty}\left(\bar{g}_{s, \alpha}(A)\right)^{1 / s} \geq \frac{1}{\underline{g}_{\infty, \alpha}(A)} \quad \text { and } \quad \liminf _{s \rightarrow \infty}\left(\underline{g}_{s, \alpha}(A)\right)^{1 / s} \geq \frac{1}{\bar{g}_{\infty, \alpha}(A)} .
$$

Upper estimates. Let, for every $N(\geq 2)$ fixed, $X_{N}=\left\{x, x_{1}, \ldots, x_{N-1}\right\} \subset \mathbf{R}^{d^{\prime}}$ be such that $a:=\delta\left(X_{N}\right)>0$ and for every $k \in \mathbf{N}$ let $M_{k}$ be the set of points from $X_{N}$ contained in $B(x, a(k+1))$ but not in $B(x, a k)$, where $B(x, r)$ is the open ball in $\mathbf{R}^{d^{\prime}}$ centered at $x$ with radius $r$. Then, from a volume argument,

$$
\# M_{k} \cdot \mathcal{L}_{d^{\prime}}[B(0, a / 2)] \leq \mathcal{L}_{d^{\prime}}[B(x, a(k+3 / 2)) \backslash B(x, a(k-1 / 2))],
$$

and so $\# M_{k} \leq(2 k+3)^{d^{\prime}}-(2 k-1)^{d^{\prime}} \leq 4 d^{\prime}(2 k+3)^{d^{\prime}-1}$. Hence,

$$
\begin{aligned}
P_{s}\left(x, X_{N}\right) & :=\sum_{i=1}^{N-1} \frac{1}{\left|x-x_{i}\right|^{s}}=\sum_{k=1}^{\infty} \sum_{x_{i} \in M_{k}} \frac{1}{\left|x-x_{i}\right|^{s}} \\
& \leq \sum_{k=1}^{\infty} \frac{\# M_{k}}{a^{s} k^{s}} \leq \frac{4 d^{\prime}}{a^{s}} \sum_{k=1}^{\infty} \frac{(2 k+3)^{d^{\prime}-1}}{k^{s}} \leq \frac{\eta_{s}}{a^{s}}, \quad s>d^{\prime},
\end{aligned}
$$

where $\eta_{s}:=\theta_{d^{\prime}} \zeta\left(s-d^{\prime}+1\right)$ and $\theta_{d^{\prime}}$ is a constant depending only on $d^{\prime}$.

Now let $\bar{\omega}_{N}:=\left\{\bar{x}_{1, N}, \ldots, \bar{x}_{N, N}\right\}$ be a best-packing $N$-point configuration on $A$; that is, $\delta\left(\bar{\omega}_{N}\right)=\delta_{N}(A)$. Then, using the above estimate, for $s>d^{\prime}$ we get

$$
\mathcal{E}_{s}(A, N) \leq E_{s}\left(\bar{\omega}_{N}\right)=\sum_{i=1}^{N} P_{s}\left(\bar{x}_{i, N}, \bar{\omega}_{N}\right) \leq \frac{\eta_{s} N}{\left(\delta_{N}(A)\right)^{s}} .
$$

Hence, for $s>d^{\prime}$ we have

$$
\bar{g}_{s, \alpha}(A) \leq \limsup _{N \rightarrow \infty} \frac{\eta_{s}}{\left(\delta_{N}(A) \cdot N^{1 / \alpha}\right)^{s}}=\frac{\eta_{s}}{\left(\underline{g}_{\infty, \alpha}(A)\right)^{s}}, \quad \underline{g}_{s, \alpha}(A) \leq \frac{\eta_{s}}{\left(\bar{g}_{\infty, \alpha}(A)\right)^{s}} .
$$

Then, since $\eta_{s}^{1 / s} \rightarrow 1$ as $s \rightarrow \infty$, we have

$$
\limsup _{s \rightarrow \infty}\left(\bar{g}_{s, \alpha}(A)\right)^{1 / s} \leq \frac{1}{\underline{g}_{\infty, \alpha}(A)}, \quad \limsup _{s \rightarrow \infty}\left(\underline{g}_{s, \alpha}(A)\right)^{1 / s} \leq \frac{1}{\bar{g}_{\infty, \alpha}(A)} .
$$


Inequalities (3.4) and (3.6) yield relations (3.1) and Proposition 3.1 is proved.

Proof of Theorem 2.3. Using (2.1) and Proposition 3.2 we get

$$
\lim _{s \rightarrow \infty} C_{s, d}^{1 / s}=\lim _{s \rightarrow \infty} g_{s, d}\left([0,1]^{d}\right)^{1 / s}=\frac{1}{g_{\infty, d}\left([0,1]^{d}\right)}=\frac{1}{C_{\infty, d}} .
$$

Proof of Theorem 2.2. Taking into account Theorem 2.1, Proposition 3.2 and Theorem 2.3. we get equation (2.3):

$$
g_{\infty, d}(A)=\left(\lim _{s \rightarrow \infty}\left(g_{s, d}(A)\right)^{1 / s}\right)^{-1}=\lim _{s \rightarrow \infty} \frac{\mathcal{H}_{d}(A)^{1 / d}}{C_{s, d}^{1 / s}}=C_{\infty, d} \mathcal{H}_{d}(A)^{1 / d} .
$$

Now suppose that $A$ is $d$-rectifiable with $\mathcal{H}_{d}(A)>0$, and $\left\{\bar{\omega}_{N}\right\}_{N=2}^{\infty}$ is a sequence of best-packing configurations on $A$ such that $\# \bar{\omega}_{N}=N$. To show that $\left\{\bar{\omega}_{N}\right\}_{N=2}^{\infty}$ is asymptotically uniformly distributed on $A$, choose any subset $B \subset A$ whose boundary relative to $A$ has $\mathcal{H}_{d}$-measure zero. Let $\bar{B}$ be the closure of the set $B$.

Set $p_{N}:=\#\left(\bar{\omega}_{N} \cap B\right)$ and let $\mathcal{N} \subset \mathbf{N}$ be an infinite subset such that the limit

$$
p(\mathcal{N}):=\lim _{\mathcal{N} \ni N \rightarrow \infty} \frac{p_{N}}{N}
$$

exists. If $p(\mathcal{N})>0$, then for sufficiently large $N \in \mathcal{N}$ we get

$$
\delta_{N}(A)=\delta\left(\bar{\omega}_{N}\right) \leq \delta\left(\bar{\omega}_{N} \cap B\right) \leq \delta_{p_{N}}(B) \leq \delta_{p_{N}}(\bar{B}) .
$$

Since $\bar{B}$ is a closed $d$-rectifiable set and $\mathcal{H}_{d}(\bar{B})=\mathcal{H}_{d}(B)$, using (2.3), we have

$$
p(\mathcal{N}) \leq \lim _{\mathcal{N} \ni N \rightarrow \infty} \frac{\delta_{p_{N}}(\bar{B})^{d} \cdot p_{N}}{\delta_{N}(A)^{d} \cdot N}=\left(\frac{g_{\infty, d}(\bar{B})}{g_{\infty, d}(A)}\right)^{d}=\frac{\mathcal{H}_{d}(B)}{\mathcal{H}_{d}(A)} .
$$

If $p(\mathcal{N})=0$, then the inequality $p(\mathcal{N}) \leq \mathcal{H}_{d}(B) / \mathcal{H}_{d}(A)$ is trivial. Thus,

$$
\limsup _{N \rightarrow \infty} \frac{p_{N}}{N} \leq \mathcal{H}_{d}(B) / \mathcal{H}_{d}(A) .
$$

Next, let $q_{N}:=\#\left(\bar{\omega}_{N} \cap(A \backslash B)\right)$. Since the boundary of $A \backslash B$ relative to $A$ also has $\mathcal{H}_{d}$-measure zero, using the same argument we can write

$$
\limsup _{N \rightarrow \infty} \frac{q_{N}}{N} \leq \frac{\mathcal{H}_{d}(A \backslash B)}{\mathcal{H}_{d}(A)}
$$

which implies that $\liminf _{N \rightarrow \infty} p_{N} / N \geq \mathcal{H}_{d}(B) / \mathcal{H}_{d}(A)$. This shows that (1.10) holds.

To prove (2.4) we will need the following lemma. Denote $\mu_{d^{\prime}}:=\mathcal{L}_{d^{\prime}}(B(0,2))$.

Lemma 3.4. Let $0<\alpha \leq d^{\prime}, G$ and $F$ be two sets in $\mathbf{R}^{d^{\prime}}$ and assume that for some positive numbers $c, \gamma$ and $\rho<\left(\gamma / \mu_{d^{\prime}}\right)^{1 / \alpha}$ there holds $\mathcal{L}_{d^{\prime}}[G(\rho) \backslash F((c+1) \rho)]>$ $\gamma \rho^{d^{\prime}-\alpha}$. Then for $N=\left\lfloor\gamma /\left(\mu_{d^{\prime}} \rho^{\alpha}\right)\right\rfloor+1$ we have $\delta_{N}(G \backslash F(c \rho)) \geq \rho$.

Proof. Let $k \in \mathbf{N} \cup\{0\}$ be the largest number of pairwise disjoint balls of radius $\rho / 2$ centered at points of $G \backslash F(c \rho)$. We just need to show that $k>\gamma /\left(\mu_{d^{\prime}} \rho^{\alpha}\right)$. Assume the contrary. Choose points $x_{1}, \ldots, x_{k} \in G \backslash F(c \rho)$ such that $\left|x_{i}-x_{j}\right| \geq \rho$, $1 \leq i \neq j \leq k$. Then

$$
\mathcal{L}_{d^{\prime}}\left(\bigcup_{i=1}^{k} B\left(x_{i}, 2 \rho\right)\right) \leq k \mu_{d^{\prime}} \rho^{d^{\prime}} \leq \gamma \rho^{d^{\prime}-\alpha}<\mathcal{L}_{d^{\prime}}[G(\rho) \backslash F((c+1) \rho)] .
$$

This means that there is a point $y \in G(\rho) \backslash F((c+1) \rho)$ such that $\left|y-x_{i}\right| \geq 2 \rho$, $i=1, \ldots, k$. Also, there exists a point $x_{k+1} \in G$ such that $\left|y-x_{k+1}\right|<\rho$. Hence, 
$\operatorname{dist}\left(x_{k+1}, F\right) \geq c \rho$. Thus, $x_{k+1} \in G \backslash F(c \rho)$ and $\left|x_{k+1}-x_{i}\right|>\rho, i=1, \ldots, k$, and so we have $k+1$ pairwise disjoint balls of radius $\rho / 2$ centered at points of $G \backslash F(c \rho)$ which contradicts to the maximality of $k$. Lemma 3.4 is proved.

Another fact needed to show (2.4) is the left inequality in (2.8). We can assume that $\overline{\mathcal{M}}_{\alpha}(A)>0$. Choose any $0<M<\overline{\mathcal{M}}_{\alpha}(A)$. Then there is a sequence $\left\{r_{m}\right\}_{m=1}^{\infty}, r_{m} \searrow 0, m \rightarrow \infty$, such that $\mathcal{L}_{d^{\prime}}\left(A\left(r_{m}\right)\right)>M \beta_{d^{\prime}-\alpha} r_{m}^{d^{\prime}-\alpha}, \quad m \in \mathbf{N}$. By Lemma 3.4 (with $F=\emptyset$ ) for the sequence $N_{m}:=\left\lfloor M \beta_{d^{\prime}-\alpha} /\left(\mu_{d^{\prime}} r_{m}^{\alpha}\right)\right\rfloor+1, m \in \mathbf{N}$, we have $\delta_{N_{m}}(A) \geq r_{m} \geq\left(M \beta_{d^{\prime}-\alpha} /\left(\mu_{d^{\prime}} N_{m}\right)\right)^{1 / \alpha}$ for sufficiently large $m$. Hence, $\bar{g}_{\infty, \alpha}(A) \geq\left(M \beta_{d^{\prime}-\alpha} / \mu_{d^{\prime}}\right)^{1 / \alpha}$. Letting $M \rightarrow \overline{\mathcal{M}}_{\alpha}(A)$, gives the lower estimate in (2.8).

Proof of inequality (2.4). In the case $\mathcal{H}_{d}(A)=0$ we have $\overline{\mathcal{M}}_{d}(A)>0$ and by the left inequality in (2.8) there holds $\bar{g}_{\infty, d}(A)>0=C_{\infty, d} \mathcal{H}_{d}(A)^{1 / d}$. Assume that $\mathcal{H}_{d}(A)>0$ and set $d^{\prime \prime}=d^{\prime}-d$. Let $c_{0} \in(0,1)$ be such that $\left(c_{0}+1\right)^{d^{\prime \prime}} \mathcal{H}_{d}(A)<$ $\overline{\mathcal{M}}_{d}(A)$ and $M_{1}, M_{2}>0$ be such numbers that

$$
\left(c_{0}+1\right)^{d^{\prime \prime}} \mathcal{H}_{d}(A)<\left(c_{0}+1\right)^{d^{\prime \prime}} M_{1}<M_{2}<\overline{\mathcal{M}}_{d}(A) .
$$

Choose any $\epsilon \in(0,1)$ and a $d$-rectifiable compact subset $K_{\epsilon} \subset A$ such that $\mathcal{H}_{d}\left(K_{\epsilon}\right)>\mathcal{H}_{d}(A)(1-\epsilon)$. By definition (1.8) there is a sequence of positive numbers $\left\{r_{m}\right\}_{m=1}^{\infty}, r_{m} \searrow 0, m \rightarrow \infty$, such that $\mathcal{L}_{d^{\prime}}\left(A\left(r_{m}\right)\right)>M_{2} \beta_{d^{\prime \prime}} \cdot r_{m}^{d^{\prime \prime}}, m \in \mathbf{N}$. By (1.9) we have $\mathcal{M}_{d}\left(K_{\epsilon}\right)=\mathcal{H}_{d}\left(K_{\epsilon}\right)<M_{1}$. Then, for sufficiently large $m$

$$
\mathcal{L}_{d^{\prime}}\left[K_{\epsilon}\left(\left(c_{0}+1\right) r_{m}\right)\right]<M_{1} \beta_{d^{\prime \prime}} \cdot\left(c_{0}+1\right)^{d^{\prime \prime}} r_{m}^{d^{\prime \prime}}
$$

and hence,

$$
\mathcal{L}_{d^{\prime}}\left[A\left(r_{m}\right) \backslash K_{\epsilon}\left(\left(c_{0}+1\right) r_{m}\right)\right]>\left(M_{2}-\left(c_{0}+1\right)^{d^{\prime \prime}} M_{1}\right) \beta_{d^{\prime \prime}} \cdot r_{m}^{d^{\prime \prime}} .
$$

By Lemma 3.4 with $\alpha=d$, there is a constant $\nu_{1}>0$ independent of $m$ and $\epsilon$, such that for $k_{m}=\left\lfloor\nu_{1} / r_{m}^{d}\right\rfloor+1$ and $m$ sufficiently large we have $\delta_{k_{m}}\left(A \backslash K_{\epsilon}\left(c_{0} r_{m}\right)\right) \geq r_{m}$. Let $X_{m} \subset A \backslash K_{\epsilon}\left(c_{0} r_{m}\right)$ be a best-packing collection of $k_{m}$ points.

Set $\nu:=C_{\infty, d} \mathcal{H}_{d}(A)^{1 / d}$. By (2.3) and the choice of $K_{\epsilon}$, for sufficiently large $N$, we have $\delta_{N}\left(K_{\epsilon}\right)>\nu(1-\epsilon)^{1 / d} N^{-1 / d}$. Choose $N_{m}$ to be the largest integer such that $\nu(1-\epsilon)^{1 / d} N_{m}^{-1 / d} \geq c_{0} r_{m}$ and denote by $Y_{m}$ the best-packing collection of $N_{m}$ points on $K_{\epsilon}$. Since $\operatorname{dist}\left(X_{m}, K_{\epsilon}\right) \geq c_{0} r_{m}$, we have that $\delta\left(X_{m} \cup Y_{m}\right) \geq c_{0} r_{m}$ for $m$ sufficiently large. Hence,

$$
\begin{gathered}
\bar{g}_{\infty, d}(A) \geq \limsup _{m \rightarrow \infty} \delta_{k_{m}+N_{m}}(A)\left(k_{m}+N_{m}\right)^{1 / d} \\
\geq \limsup _{m \rightarrow \infty} c_{0} r_{m}\left(\frac{\nu_{1}}{r_{m}^{d}}+\frac{\nu^{d}(1-\epsilon)}{c_{0}^{d} r_{m}^{d}}-1\right)^{1 / d}=\left(c_{0}^{d} \nu_{1}+\nu^{d}(1-\epsilon)\right)^{1 / d} .
\end{gathered}
$$

Letting $\epsilon \rightarrow 0$, we get

$$
\bar{g}_{\infty, d}(A) \geq\left(c_{0}^{d} \nu_{1}+\nu^{d}\right)^{1 / d}>\nu=C_{\infty, d} \mathcal{H}_{d}(A)^{1 / d} .
$$

This completes the proof of Theorem 2.2

Proof of Proposition 2.4. Using Proposition 3.1, Theorem 2.3, and inequality (2.4) we have

$$
\lim _{s \rightarrow \infty}\left(\frac{\underline{g}_{s, d}(A)}{C_{s, d} \mathcal{H}_{d}(A)^{-s / d}}\right)^{1 / s}=\frac{C_{\infty, d} \mathcal{H}_{d}(A)^{1 / d}}{\bar{g}_{\infty, d}(A)}<1
$$


and the required inequality follows for sufficiently large $s$.

Proof of Proposition 2.5. We only need to prove (2.7) and (2.8) since the upper estimates in (2.5) and (2.6) will follow from (3.5) and the lower estimates in (2.7) and (2.8). Analogously, the lower estimates in (2.5) and (2.6) are obtained from the upper estimates in (2.7) and (2.8), using (3.2) or (3.3) with $\epsilon$ equal, say $1 / 2$. We remark that (3.2), (3.3) and (3.5) hold for any infinite set $A$.

Since we do not look for sharp constants, redefine

$$
\underline{\mathcal{M}}_{\alpha}(A):=\liminf _{r \rightarrow 0^{+}} \mathcal{L}_{d^{\prime}}(A(r)) / r^{d^{\prime}-\alpha} \text { and } \quad \overline{\mathcal{M}}_{\alpha}(A):=\limsup _{r \rightarrow 0^{+}} \mathcal{L}_{d^{\prime}}(A(r)) / r^{d^{\prime}-\alpha} \text {. }
$$

To show the lower estimate in (2.7), assume that $\underline{\mathcal{M}}_{\alpha}(A)>0$ (otherwise it is trivial). Pick any $0<M<\underline{\mathcal{M}}_{\alpha}(A)$ and set $r_{N}:=\left(M /\left(\mu_{d^{\prime}} N\right)\right)^{1 / \alpha}$. Then, for $N$ sufficiently large $\mathcal{L}_{d^{\prime}}\left(A\left(r_{N}\right)\right)>M r_{N}^{d^{\prime}-\alpha}$. By Lemma 3.4 (with $F=\emptyset$ ), for $k_{N}=\left\lfloor M /\left(\mu_{d^{\prime}} r_{N}^{\alpha}\right)\right\rfloor+$ $1\left(k_{N}\right.$ will be greater that $\left.N\right)$ we have $\delta_{N}(A) \geq \delta_{k_{N}}(A) \geq r_{N}=\left(M /\left(\mu_{d^{\prime}} N\right)\right)^{1 / \alpha}$ for sufficiently large $N$. Hence, $\underline{g}_{\infty, \alpha}(A) \geq \mu_{d^{\prime}}^{-1 / \alpha} M^{1 / \alpha}$. Letting $M \rightarrow \underline{\mathcal{M}}_{\alpha}(A)$, we get the lower estimate in (2.7). We need the following lemma for the upper estimate.

Lemma 3.5. Let $0<\alpha \leq d^{\prime}, A \neq \emptyset$ be a set in $\mathbf{R}^{d^{\prime}}$ and, for some positive numbers $\gamma$ and $\rho<\left(\gamma / \beta_{d^{\prime}}\right)^{1 / \alpha}$, assume that there holds $\mathcal{L}_{d^{\prime}}(A(\rho))<\gamma \rho^{d^{\prime}-\alpha}$. Then for any $N>\gamma /\left(\beta_{d^{\prime}} \rho^{\alpha}\right)$ we have $\delta_{N}(A) \leq 2 \rho$.

Proof. Suppose $k \geq 2$ is an integer such that $\delta_{k}(A)>2 \rho$, and let $x_{1}, \ldots, x_{k} \in A$ be a collection of distinct points with separation at least $2 \rho$. Then

$$
\mathcal{L}_{d^{\prime}}\left(\bigcup_{i=1}^{k} B\left(x_{i}, \rho\right)\right)=k \beta_{d^{\prime}} \rho^{d^{\prime}} \leq \mathcal{L}_{d^{\prime}}(A(\rho))<\gamma \rho^{d^{\prime}-\alpha} .
$$

Hence, $k \leq \gamma /\left(\beta_{d^{\prime}} \rho^{\alpha}\right)$, and so for any $N>\gamma /\left(\beta_{d^{\prime}} \rho^{\alpha}\right)$ we have $\delta_{N}(A) \leq 2 \rho$, which proves the lemma.

To get the upper estimate in (2.7), we can assume that $\underline{\mathcal{M}}_{\alpha}(A)<\infty$. Choose any $M>\underline{\mathcal{M}}_{\alpha}(A)$. There is a sequence of positive numbers $\left\{r_{m}\right\}_{m=1}^{\infty}, r_{m} \searrow 0$, $m \rightarrow \infty$, such that $\mathcal{L}_{d^{\prime}}\left(A\left(r_{m}\right)\right)<M r_{m}^{d^{\prime}-\alpha}, \quad m \in \mathbf{N}$. Set $N_{m}:=\left\lfloor M /\left(\beta_{d^{\prime}} r_{m}^{\alpha}\right)\right\rfloor+1$. By Lemma 3.5 we have $\delta_{N_{m}}(A) \leq 2 r_{m}$ for sufficiently large $m$. Consequently,

$$
\underline{g}_{\infty, \alpha}(A) \leq \liminf _{m \rightarrow \infty} \delta_{N_{m}}(A) N_{m}^{1 / \alpha} \leq \liminf _{m \rightarrow \infty} 2 r_{m} N_{m}^{1 / \alpha}=2 \beta_{d^{\prime}}^{-1 / \alpha} M^{1 / \alpha} .
$$

Letting $M \rightarrow \underline{\mathcal{M}}_{\alpha}(A)$ completes the proof of (2.7).

The left inequality in (2.8) was shown before the proof of inequality (2.4). Thus, it remains to prove the right inequality in (2.8) for the case $\overline{\mathcal{M}}_{\alpha}(A)<\infty$. Pick any $M>\overline{\mathcal{M}}_{\alpha}(A)$ and let $r_{N}:=\left(M /\left(\beta_{d^{\prime}}(N-1)\right)\right)^{1 / \alpha}, N \geq 2$. Then $\mathcal{L}_{d^{\prime}}\left(A\left(r_{N}\right)\right)<$ $M r_{N}^{d^{\prime}-\alpha}$ for $N$ sufficiently large. Since, $N>M /\left(\beta_{d^{\prime}} r_{N}^{\alpha}\right)$, by Lemma 3.5 we get $\delta_{N}(A) \leq 2 r_{N}=2\left(M /\left(\beta_{d^{\prime}}(N-1)\right)\right)^{1 / \alpha}$. Hence, $\bar{g}_{\infty, \alpha}(A) \leq 2 \beta_{d^{\prime}}^{-1 / \alpha} M^{1 / \alpha}$. Letting $M \rightarrow \overline{\mathcal{M}}_{\alpha}(A)$ completes the proof of (2.8) and Proposition 2.5.

Proof of Proposition 2.6. It was shown in [14] (see also [18, Theorem 4.14]) that for any set $K \in \mathcal{K}$ there are constants $c_{1}, c_{2}>0$ such that

$$
c_{1} r^{\lambda} \leq \mathcal{H}_{\lambda}(K \cap B(x, r)) \leq c_{2} r^{\lambda}, \quad x \in K, \quad 0<r<1 .
$$

Using an argument analogous to the proof of Lemma 3.5. one can show that $\bar{g}_{\infty, \lambda}(K)<\infty$. Since for any set $K \in \mathcal{K}$ we have $\underline{\mathcal{M}}_{\lambda}(K) \geq C \mathcal{H}_{\lambda}(K)>0$ with $C>0$ independent of $K$ (cf. e.g. [18, p. 79]), by (2.7) we have $\underline{g}_{\infty, \lambda}(K)>0$. 
Assume that $g_{\infty, \lambda}(K)$ exists. Let $S_{1}, \ldots, S_{p}: \mathbf{R}^{d^{\prime}} \rightarrow \mathbf{R}^{d^{\prime}}$ be the similitudes with the same contraction coefficient $\sigma \in(0,1)$ such that relations (2.9) hold. Set $h:=\min _{i \neq j} \operatorname{dist}\left(S_{i}(K), S_{j}(K)\right)$ and choose $k \in \mathbf{N}$ so that $\delta_{k}(K)<h$.

Let $m \in \mathbf{N}$ and for $\mathbf{i}=\left(i_{1}, \ldots, i_{m}\right) \in\{1, \ldots, p\}^{m}=: Z_{p}^{m}$ put $F_{\mathbf{i}}:=S_{i_{1}} \circ \cdots \circ S_{i_{m}}$. Then

$$
\operatorname{dist}\left(F_{\mathbf{i}}(K), F_{\mathbf{j}}(K)\right) \geq h \sigma^{m-1}>\sigma^{m-1} \delta_{k}(K), \quad \mathbf{i} \neq \mathbf{j},
$$

and $\bigcup_{\mathbf{i} \in Z_{p}^{m}} F_{\mathbf{i}}(K)=K$. Let $\bar{\omega}_{k} \subset K$ be a collection of $k$ points such that $\delta\left(\bar{\omega}_{k}\right)=$ $\delta_{k}(K)$, and $\omega_{m}:=\bigcup_{\mathbf{i} \in Z_{p}^{m}} F_{\mathbf{i}}\left(\bar{\omega}_{k}\right)$. In view of (3.9), it is not difficult to see that $\delta_{k p^{m}}(K) \geq \delta\left(\omega_{m}\right)=\sigma^{m} \delta_{k}(K)$. On the other hand, from any collection of $c_{m}:=$ $(k-1) p^{m}+1$ points on $K$ at least $k$ must belong to the same $F_{\mathbf{i}}(K)$, and hence, $\delta_{c_{m}}(K) \leq \sigma^{m} \delta_{k}(K)$. Since $c_{m} \leq k p^{m}$, we have $\delta_{k p^{m}}(K)=\delta_{c_{m}}(K)$ and

$$
\begin{gathered}
g_{\infty, \lambda}(K)=\lim _{m \rightarrow \infty} \delta_{k p^{m}}(K)\left(k p^{m}\right)^{1 / \lambda}=\lim _{m \rightarrow \infty} \delta_{c_{m}}(K)\left(k p^{m}\right)^{1 / \lambda} \\
=g_{\infty, \lambda}(K) \lim _{m \rightarrow \infty}\left(k p^{m} / c_{m}\right)^{1 / \lambda}=g_{\infty, \lambda}(K)(k /(k-1))^{1 / \lambda},
\end{gathered}
$$

which contradicts the finiteness and positiveness of $g_{\infty, \lambda}(K)$. Hence, $0<\underline{g}_{\infty, \lambda}(K)<$ $\bar{g}_{\infty, \lambda}(K)<\infty$. Taking into account Propositions 3.1] and 3.3. we get Proposition 2.6 .

\section{REFERENCES}

1. N.N. Andreev, On positions of points with minimal energy, Papers of the V.A. Steklov Math. Institute 219 (1997), 27-31.

2. K. Böröczky Jr., Finite Packing and Covering, Cambridge University Press, 2004. MR2078625 (2005g:52045)

3. S.V. Borodachov, D.P. Hardin, E.B. Saff, Asymptotics for discrete weighted minimal Riesz energy problems on rectifiable sets, Trans. Amer. Math. Soc., to appear.

4. J.H. Conway, N.J.A. Sloane, Sphere Packings, Lattices and Groups, Springer Verlag, New York: 3rd ed., 1999. MR1662447(2000b:11077)

5. H. Federer, Geometric measure theory, Springer-Verlag, Berlin-Heidelberg-New York, 1969. MR0257325 (41:1976)

6. L. Fejes Toth, Lagerungen in der Ebene auf der Kugel und im Raum, Springer Verlag, BerlinGöttingen-Heidelberg, 1953. MR0057566 (15:248b)

7. L. Fejes Toth, Regular figures, Pergamon Press, The Macmillan Co., New York, 1964. MR0165423 (29:2705)

8. S. Graf, H. Luschgy, Foundations of quantization for probability distributions, Lect. Notes in Math., vol. 1730, Springer, 2000. MR1764176 (2001m:60043)

9. P. M. Gruber, Optimum quantization and its applications, Adv. Math. 186 (2004), no. 2, 456-497. MR2073915 (2005e:94060)

10. T.C. Hales, A proof of the Kepler conjecture, Ann. of Math. (2) 162 (2005), no. 3, 1065-1185. MR2179728 (2006g:52029)

11. D.P. Hardin, E.B. Saff, Discretizing manifolds via minimum energy points, Notices Amer. Math. Soc. 51 (2004), no. 10, 1186-1194. MR2104914 (2006a:41049)

12. D.P. Hardin, E.B. Saff, Minimal Riesz energy point configurations for rectifiable $d$ dimensional manifolds, Adv. Math. 193 (2005), 174-204. MR.2132763 (2005m:49006)

13. W. Habicht, B. L. van der Waerden, Lagerung von Punkten auf der Kugel, Math. Ann. 123 (1951), 223-234. MR0042730(13:154d)

14. J.E. Hutchinson, Fractals and self-similarity, Indiana Univ. Math. J. 30 (1981), 743-747. MR625600(82h:49026)

15. A.V. Kolushov, V.A. Yudin, Extremal dispositions of points on the sphere, Anal. Math. 23 (1997) no. 1, 25-34. MR.1630001 (99f:41039)

16. N.S. Landkof, Foundations of modern potential theory, Springer-Verlag, Berlin-HeidelbergNew York, 1972. MR0350027 (50:2520) 
17. A. Martinez-Finkelshtein, V. Maymeskul, E. Rakhmanov, E.B. Saff, Asymptotics for minimal discrete Riesz energy on curves in $\mathbf{R}^{d}$, Canad. J. Math. 56 (2004), 529-552. MR2057285 (2005a:31010)

18. P. Mattila, Geometry of sets and measures in Euclidean spaces. Fractals and Rectifiability, Cambridge Univ. Press, 1995. MR,1333890 (96h:28006)

19. C.A. Rogers, Packing and covering, Cambridge Tracts in Mathematics and Mathematical Physics, vol. 54, Cambridge University Press, New York, 1964. MR0172183 (30:2405)

20. J.J. Thomson, On the Structure of the Atom: an Investigation of the Stability and Periods of Oscillation of a number of Corpuscles arranged at equal intervals around the Circumference of a Circle; with Application of the results to the Theory of Atomic Structure, Philosophical Magazine, Sixth Series, 7 (1904), 237-265.

21. B. L. van der Waerden, Punkte auf der Kugel. Drei Zusätze, Math. Ann. 125 (1952), 213-222. MR0050912 (14:401c)

22. V.A. Yudin, The minimum of potential energy of a system of point charges, Discrete Math. Appl. Vol. 3, No. 1 (1993), 75-81. MR1181534 (93f:31008)

School of Mathematics, Georgia Institute of Technology, Atlanta, Georgia, 30332

E-mail address: borodasv@math.gatech.edu

Department of Mathematics, Vanderbilt University, Nashville, Tennessee 37240

E-mail address: doug.hardin@vanderbilt.edu

Department of Mathematics, Vanderbilt University, Nashville, Tennessee 37240

E-mail address: Edward.B.Saff@Vanderbilt.edu 